\title{
A Non-Cooperative Power Control Game for Multi-Carrier CDMA Systems
}

\author{
Farhad Meshkati, Mung Chiang, Stuart C. Schwartz, and H. Vincent Poor \\ Department of Electrical Engineering, Princeton University \\ Princeton, NJ 08544 USA \\ \{meshkati, chiangm, stuart, poor\}@princeton.edu \\ Narayan B. Mandayam \\ WINLAB, Rutgers University \\ Piscataway, NJ 08854 USA \\ narayan@winlab.rutgers.edu
}

\begin{abstract}
In this work, a non-cooperative power control game for multi-carrier CDMA systems is proposed. In the proposed game, each user needs to decide how much power to transmit over each carrier to maximize its overall utility. The utility function considered here measures the number of reliable bits transmitted per joule of energy consumed. It is shown that the user's utility is maximized when the user transmits only on the carrier with the best "effective channel". The existence and uniqueness of Nash equilibrium for the proposed game are investigated and the properties of equilibrium are studied. Also, an iterative and distributed algorithm for reaching the equilibrium (if it exists) is presented. It is shown that the proposed approach results in a significant improvement in the total utility achieved at equilibrium compared to the case in which each user maximizes its utility over each carrier independently.
\end{abstract}

\section{INTRODUCTION}

Power control is used for resource allocation and interference management in both the uplink and downlink of code division multiple access (CDMA) systems. In the uplink, the purpose of power control is for each user to transmit enough power so that it can achieve the required quality of service (QoS) at the uplink receiver without causing unnecessary interference to other users in the system. Game theory has been used as an effective tool to study power control for data networks (see for example [1-6]). Taking this approach, it is shown in [1] and [3] that Nash equilibrium is achieved when all the users aim for a target SIR, $\gamma^{*}$. This is true even when the matched filter is replaced by the decorrelator or the minimum mean square error (MMSE) receiver as shown in [4]. Nash equilibrium is a set of strategies such that no user can unilaterally improve its own utility (which is a measure of user satisfaction) given the strategies taken by other users. Extension of the above results to multiantenna systems are also presented in [5].

Multi-carrier CDMA, which combines the benefits of orthogonal frequency division multiplexing (OFDM) with those of CDMA, is considered to be a potential candidate for next generation high data-rate wireless systems (see [7]). In this work, we consider an orthogonal multi-carrier DS-CDMA system and propose a non-cooperative power control game in

This research was supported by the National Science Foundation under Grants ANI-03-38807, CNS-04-17603 and CCR-04-40443. which each user tries to choose its transmit power over each carrier to maximize its overall utility. For the proposed game, we will address the following questions. When does a Nash equilibrium exist? What kind of carrier allocations among the competing users will occur at a Nash equilibrium? Will there be an even spread of usage of the carriers among users? How does this approach compare with an approach in which each user simply optimizes over each carrier independently?

The rest of this paper is organized as follows. In Section we provide some background for this work by discussing the power control game for the single-carrier case. The power control game for multi-carrier systems is presented in Section III The Nash equilibrium and its existence for the proposed game are discussed in Section [V] and a distributed algorithm for reaching the equilibrium (if it exists) is presented in Section $\nabla$ The case of two-carrier systems is studied in more details in Section VI Numerical results and conclusions are given in Sections VII and VIII respectively. Our focus throughout this work is on the uplink, where user terminals transmit to a common concentration point such as a cellular base station.

\section{BACKGROUND}

Let us first look at the power control game with a single carrier. To pose the power control problem as a non-cooperative game, we first need to define a utility function suitable for data applications. Most data applications are sensitive to error but they can tolerate delay. It is clear that a higher signal to interference plus noise ratio (SIR) level at the output of the receiver will result in a lower bit error rate and hence higher throughput. However, achieving a high SIR level requires the user terminal to transmit at a high power which in turn results in low battery life. In [1], the utility function of a user is defined as the ratio of its throughput to its transmit power, i.e.

$$
u_{k}=\frac{T_{k}}{p_{k}} .
$$

Throughput is the net number of information bits that are transmitted without error per unit time (sometimes referred to as goodput). It can be expressed as

$$
T_{k}=\frac{L}{M} R_{k} f\left(\gamma_{k}\right),
$$


where $L$ and $M$ are the number of information bits and the total number of bits in a packet, respectively. $R_{k}$ and $\gamma_{k}$ are the transmission rate and the SIR for the $k^{\text {th }}$ user, respectively; and $f\left(\gamma_{k}\right)$ is the efficiency function representing the packet success rate (PSR), i.e. the probability that a packet is received without an error. Our assumption is that if a packet has one or more bit errors, it will be retransmitted. The efficiency function, $f\left(\gamma_{k}\right)$, is assumed to be increasing and S-shaped (sigmoidal) with $f(\infty)=1$. We also require that $f(0)=0$ to ensure that $u_{k}=0$ when $p_{k}=0$. These assumptions are valid in many practical systems. It should be noted that the throughput $T_{k}$ in (2) could be replaced with any increasing concave function as long as we make sure that $u_{k}=0$ when $p_{k}=0$. A more detailed discussion of the efficiency function can be found in [6] and [8]. Note that for a sigmoidal efficiency function, the utility function in (1) is quasiconcave in user's transmit power. This is also true if the throughput in (2) is replaced with an increasing concave function.

Based on (1) and (2), the utility function for user $k$ can be written as

$$
u_{k}=\frac{L}{M} R_{k} \frac{f\left(\gamma_{k}\right)}{p_{k}} .
$$

This utility function, which has units of bits/Joule, captures very well the tradeoff between throughput and battery life and is particularly suitable for applications where saving power is as important as achieving a high throughput.

Power control is modeled as a non-cooperative game in which each user tries to selfishly maximize its own utility. It is shown in [3] that, when matched filters are used as the uplink receivers, if user terminals are allowed to choose only their transmit powers for maximizing their utilities, then there exists an equilibrium point at which no user can improve its utility given the power levels of other users (Nash equilibrium). The equilibrium is achieved when users' transmit powers are SIRbalanced with $\gamma^{*}$, the solution to $f(\gamma)=\gamma f^{\prime}(\gamma)$, as the output SIR. Furthermore, this equilibrium is unique.

In this work, we extend this game-theoretic approach to multi-carrier systems.

\section{The Non-Cooperative Power Control Game in MULTI-CARRIER SYSTEMS}

Let us consider the uplink of a multi-carrier DS-CDMA data network with $K$ users, $D$ carriers and processing gain $N$ (for each carrier). The carriers are assumed to be sufficiently far apart so that the (spread-spectrum) signal transmitted over each carrier does not interfere with the signals transmitted over other carriers. At the transmitter, the incoming bits for user $k$ are divided into $D$ parallel streams and each stream is spread using the spreading code of user $k$. The $D$ parallel streams are then sent over the $D$ (orthogonal) carriers. For the $\ell^{t h}$ carrier, the received signal at the uplink receiver (after chip-matched filtering and sampling) can be represented by an $N \times 1$ vector as

$$
\mathbf{r}_{\ell}=\sum_{k=1}^{K} \sqrt{p_{k \ell} h_{k \ell}} b_{k \ell} \mathbf{s}_{k}+\mathbf{w}_{\ell}
$$

where $b_{k \ell}, p_{k \ell}, h_{k \ell}$ are the $k^{t h}$ user's transmitted bit, transmit power and path gain, respectively, for the $\ell^{t h}$ frequency channel (carrier). $\mathbf{s}_{k}$ is the spreading sequence for user $k$ which is assumed to be random with unit norm; and $\mathbf{w}_{\ell}$ is the noise vector which is assumed to be Gaussian with mean $\mathbf{0}$ and covariance $\sigma^{2} \mathbf{I}$. The matched filter output SIR for the $\ell^{t h}$ carrier of the $k^{t h}$ user is, therefore, given by

$$
\gamma_{k \ell}=\frac{p_{k \ell} h_{k \ell}}{\sigma^{2}+\frac{1}{N} \sum_{j \neq k} p_{j \ell} h_{j \ell}} .
$$

We propose a non-cooperative game in which each user chooses its transmit powers over the $D$ carriers to maximize its overall utility. In other words, each user (selfishly) decides how much power to transmit over each frequency channel (carrier) to achieve the highest overall utility. Let $G_{D}=$ $\left[\mathcal{K},\left\{A_{k}^{M C}\right\},\left\{u_{k}^{M C}\right\}\right]$ denote the proposed non-cooperative game where $\mathcal{K}=\{1, \ldots, K\}$, and $A_{k}^{M C}=\left[0, P_{\max }\right]^{D}$ is the strategy set for the $k^{t h}$ user. Here, $P_{\max }$ is the maximum transmit power. Each strategy in $A_{k}^{M C}$ can be written as $\mathbf{p}_{k}=\left[p_{k}^{1}, \ldots, p_{k}^{D}\right]$. The utility function for user $k$ is defined as the ratio of the total throughput over the total transmit power for the $D$ carriers, i.e.

$$
u_{k}^{M C}=\frac{\sum_{\ell=1}^{D} T_{k \ell}}{\sum_{\ell=1}^{D} p_{k \ell}},
$$

where $T_{k \ell}$ is the throughput achieved by user $k$ over the $\ell^{t h}$ carrier, and is given by $T_{k \ell}=\frac{L}{M} R_{k} f\left(\gamma_{k \ell}\right)$. Hence, the resulting non-cooperative game can be expressed as the following maximization problem:

$$
\max _{\mathbf{p}_{k}} u_{k}^{M C}=\max _{p_{k 1}, \ldots, p_{k D}} u_{k}^{M C} \quad \text { for } \quad k=1, \ldots, K,
$$

under the constraint of non-negative powers (i.e. $p_{k \ell} \geq 0$ for all $k=1, \ldots, K$ and $\ell=1, \ldots, D)$. Without any loss of generality, if we assume equal transmission rates for all users, (7) can be expressed as

$$
\max _{p_{k 1}, \ldots, p_{k D}} \frac{\sum_{\ell=1}^{D} f\left(\gamma_{k \ell}\right)}{\sum_{\ell=1}^{D} p_{k \ell}} \quad \text { for } k=1, \ldots, K .
$$

The relationship between $\gamma_{k \ell}$ and $p_{k \ell}$ 's is given by (5).

\section{EXISTENCE OF NASH EQUILIBRIUM FOR THE PROPOSED GAME}

For the non-cooperative power control game proposed in the previous section, a Nash equilibrium is a set of power vectors, $\mathbf{p}_{1}^{*}, \ldots, \mathbf{p}_{K}^{*}$, such that no user can unilaterally improve its utility by choosing a different power vector; i.e. $\mathbf{p}_{1}^{*}, \ldots, \mathbf{p}_{K}^{*}$ is a Nash equilibrium if and only if

$$
u_{k}^{M C}\left(\mathbf{p}_{k}^{*}, \mathbf{P}_{-k}^{*}\right) \geq u_{k}^{M C}\left(\mathbf{p}_{k}, \mathbf{P}_{-k}^{*}\right) \text { for all } \mathbf{p}_{k},
$$

and for $k=1, \cdots, K$; where $\mathbf{P}_{-k}^{*}$ contains the transmit power vectors of all the users except for user $k$.

Let us define

$$
\hat{h}_{k \ell}=\frac{h_{k \ell}}{\sigma^{2}+\frac{1}{N} \sum_{j \neq k} p_{j \ell} h_{j \ell}}
$$

as the "effective channel gain" for user $k$ over the $\ell^{t h}$ carrier. Based on (5) and (10), we have $\gamma_{k \ell}=\hat{h}_{k \ell} p_{k \ell}$. 
Proposition 1: For user $k$, the utility function in 6 is maximized when

$$
p_{k \ell}= \begin{cases}p_{k}^{*} & \text { for } \quad \ell=L_{k} \\ 0 & \text { for } \quad \ell \neq L_{k}\end{cases}
$$

where $L_{k}=\arg \max _{\ell} \hat{h}_{k \ell}$ and $p_{k}^{*}=\min \left\{\frac{\gamma^{*}}{\hat{h}_{k}^{L}}, P_{\max }\right\}$, with $\gamma^{*}$ being the unique (positive) solution of $f(\gamma)=\gamma f^{\prime}(\gamma)$.

Proof: We first show that $\frac{f(\tilde{a} p)}{p}$ is maximized when $p=\gamma^{*} / \tilde{a}$. For this, we take the derivative of $\frac{f(\tilde{a} p)}{p}$ with respect to $p$ and equate it to zero. Letting $\gamma=\tilde{a} p$, we have

$$
p \frac{\partial \gamma}{\partial p} f^{\prime}(\gamma)-f(\gamma)=0
$$

Since $p \frac{\partial \gamma}{\partial p}=\gamma, p^{*}=\min \left\{\gamma^{*} / \tilde{a}, P_{\max }\right\} \operatorname{maximizes} \frac{f(\tilde{a} p)}{p}$, where $\gamma^{*}$ is the unique (positive) solution to $f(\gamma)=\gamma f^{\prime}(\gamma)$. Now, without any loss of generality, let us assume that $\hat{h}_{k 1}>\hat{h}_{k 2}>\ldots>\hat{h}_{k D}$. Then, based on the above argument we can write $\frac{f\left(\hat{h}_{k 1} p_{k 1}\right)}{p_{k 1}} \leq \frac{f\left(\hat{h}_{k 1} p_{k}^{*}\right)}{p_{k}^{*}}$ for any $p_{k 1} \geq 0$, where $p_{k}^{*}=\gamma^{*} / \hat{h}_{k 1}$. Also, because $\hat{h}_{k 1}>\hat{h}_{k 2}>\ldots>\hat{h}_{k D}$, we have $\frac{f\left(\hat{h}_{k \ell} p_{k \ell}\right)}{p_{k \ell}} \leq \frac{f\left(\hat{h}_{k 1} p_{k}^{*}\right)}{p_{k}^{*}}$ for all $p_{k \ell} \geq 0$ and $\ell=2,3, \ldots, K$. Based on the above inequalities, we can write

$$
\frac{f\left(\hat{h}_{k \ell} p_{k \ell}\right)}{f\left(\hat{h}_{k}^{1} p_{k}^{*}\right)} \leq \frac{p_{k \ell}}{p_{k}^{*}}, \quad \text { for } \ell=1,2, \ldots, D .
$$

Adding the $D$ inequalities given in 13 and rewriting the resulting inequality, we have

$$
\frac{\sum_{\ell=1}^{D} f\left(\hat{h}_{k \ell} p_{k \ell}\right)}{\sum_{\ell=1}^{D} p_{k \ell}} \leq \frac{f\left(\hat{h}_{k 1} p_{k}^{*}\right)}{p_{k}^{*}} \text { for all } p_{k 1}, \ldots, p_{k D} \geq 0 \text {. }
$$

This completes the proof.

Proposition 1 suggests that the utility for user $k$ is maximized when the user transmits only over its "best" carrier such that the achieved SIR at the output of the matched filter receiver is equal to $\gamma^{*}$. The "best" carrier is the one with the largest effective channel gain. An alternative way of interpreting Proposition 1 is that the utility for user $k$ is maximized when the user transmits only on the carrier that requires the least amount of transmit power to achieve $\gamma^{*}$ at the output of the uplink receiver. A set of power vectors, $\mathbf{p}_{1}^{*}, \ldots, \mathbf{p}_{K}^{*}$, is a Nash equilibrium if and only if they simultaneously satisfy 11 .

It should also be noted that the utility-maximizing strategy suggested by Proposition 1 is different from the waterfilling approach discussed in [9]. This is because in [9], utility is defined as the user's throughput whereas here we have defined utility as the number of bits transmitted per joule of energy consumed. Another observation is that if we use $\tilde{u}_{k}=\sum_{\ell=1}^{D} \frac{T_{k \ell}}{p_{k \ell}}$ as the utility function, then the utility for user $k$ is maximized when the user transmits on all the carriers at power levels that achieve $\gamma^{*}$ for every carrier. This is equivalent to the case in which each user maximizes its utility over each carrier independently.

Since at Nash equilibrium (if it exists), each user must transmit on one carrier only, there are exactly $D^{K}$ possibilities for an equilibrium. For example, in the case of $K=D=2$, there are 4 possibilities for Nash equilibrium:

- User 1 and user 2 both transmit on the first carrier.

- User 1 and user 2 both transmit on the second carrier.

- User 1 transmits on the first carrier and user 2 transmits on the second carrier.

- User 1 transmits on the second carrier and user 2 transmits on the first carrier.

Depending on the set of channel gains, i.e. $h_{k \ell}$ 's , the proposed power control game may have no equilibrium, a unique equilibrium, or more than one equilibrium. Let us for now assume that the processing gain is sufficiently large so that even when all $K$ users transmit on the same carrier, $\gamma^{*}$ can be achieved by all users. This is the case when $N>(K-1) \gamma^{*}$. The following proposition helps identify the Nash equilibrium (if it exists) for a given set of channel gains.

Proposition 2: The necessary condition for user $k$ to transmit on the $\ell^{\text {th }}$ carrier at equilibrium is that

$$
\frac{h_{k \ell}}{h_{k i}}>\frac{\Theta_{n(\ell)}}{\Theta_{n(i)}} \Theta_{0} \quad \text { for all } i \neq \ell
$$

where $n(i)$ is the number of users transmitting on the $i^{t h}$ carrier and

$$
\Theta_{n}=\frac{1}{1-(n-1) \frac{\gamma^{*}}{N}} \quad n=0,1, \ldots, K .
$$

In this case, $p_{k \ell}=\frac{\gamma^{*} \sigma^{2}}{h_{k \ell}} \Theta_{n(\ell)}$.

Proof: Based on Proposition 1, in order for user $k$ to transmit on carrier $\ell$ at equilibrium, we must have

$$
\hat{h}_{k \ell}>\hat{h}_{k}^{i} \text { for all } i \neq \ell \text {. }
$$

Since $n(\ell)$ users (including user $k$ ) are transmitting on the $\ell^{\text {th }}$ carrier and $n(i)$ users are transmitting on the $i^{\text {th }}$ carrier and all users have an output SIR equal to $\gamma^{*}$, we have

$$
\hat{h}_{k \ell}=\frac{h_{k \ell}}{\sigma^{2}+\frac{n(\ell)-1}{N} q_{\ell}},
$$

and

$$
\hat{h}_{k i}=\frac{h_{k i}}{\sigma^{2}+\frac{n(i)}{N} q_{i}},
$$

where $q_{\ell}=\frac{\sigma^{2} \gamma^{*}}{1-\frac{(n(\ell)-1) \gamma^{*}}{N}}$ and $q_{i}=\frac{\sigma^{2} \gamma^{*}}{1-\frac{(n(i)-1) \gamma^{*}}{N}}$ are the received powers for each user on the $\ell^{t h}$ and $i^{t^{N}}$ carriers, respectively. Now define $\Theta_{n}=\frac{1}{1-(n-1) \frac{\gamma^{*}}{N}}$ to get $q_{\ell}=\sigma^{2} \gamma^{*} \Theta_{n(\ell)}$ and $q_{i}=\sigma^{2} \gamma^{*} \Theta_{n(i)}$. Substituting $q_{\ell}$ and $q_{i}$ into (18) and 19. and taking advantage of the fact that $1+\frac{(n-1) \gamma^{*}}{N} \Theta_{n}=\Theta_{n}$ we get

$$
\hat{h}_{k \ell}=\frac{h_{k \ell}}{\Theta_{n(\ell)}}
$$

and

$$
\hat{h}_{k i}=\frac{h_{k i}}{\frac{\Theta_{n(i)}}{\Theta_{0}}} .
$$

Consequently, (15) is obtained by substituting (20) and 21) into (17). Furthermore, since $p_{k \ell} h_{k \ell}=q_{\ell}=\sigma^{2} \gamma^{*} \Theta_{n(\ell)}$, we have $p_{k \ell}=\frac{\gamma^{*} \sigma^{2}}{h_{k \ell}} \Theta_{n(\ell)}$ and this completes the proof. 


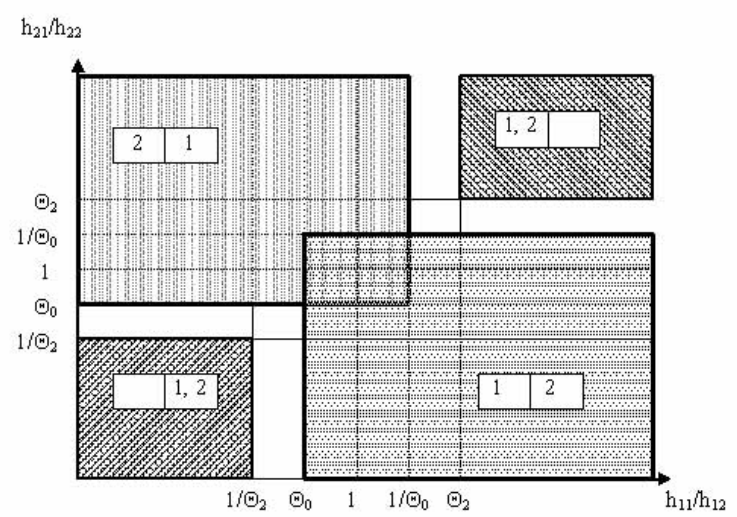

Fig. 1. Nash Equilibrium Regions for the Case of $K=2$ and $D=2$

Based on 16, when $N>(K-1) \gamma^{*}$, we have $0<\Theta_{0}<\Theta_{1}<\Theta_{2}<\ldots<\Theta_{K}$ with $\Theta_{1}=1$.

For each of the $D^{K}$ possible equilibria, the channel gains for each of the $K$ users must satisfy $D-1$ inequalities similar to 15. Furthermore, satisfying a set of $K(D-1)$ of such inequalities by the $K$ users is sufficient for existence of Nash equilibrium but the uniqueness is not guaranteed. For example, for the case of $K=D=2$, the 4 possible equilibria can be characterized as follows.

- For both users to transmit on the first carrier at equilibrium, we must have $\frac{h_{11}}{h_{12}}>\Theta_{2}$ and $\frac{h_{21}}{h_{22}}>\Theta_{2}$.

- For both users to transmit on the second carrier at equilibrium, we must have $\frac{h_{12}}{h_{11}}>\Theta_{2}$ and $\frac{h_{22}}{h_{21}}>\Theta_{2}$.

- For user 1 and user 2 to transmit on the first and second carriers, respectively, at equilibrium, we must have $\frac{h_{11}}{h_{12}}>\Theta_{0}$ and $\frac{h_{22}}{h_{21}}>\Theta_{0}$.

- For user 1 and user 2 to transmit on the second and first carriers, respectively, at equilibrium, we must have $\frac{h_{12}}{h_{11}}>\Theta_{0}$ and $\frac{h_{21}}{h_{22}}>\Theta_{0}$.

Fig. 1] shows the regions corresponding to the above four equilibria. It can be seen that for certain values of channel gains there is no Nash equilibrium and for some values of channel gains there are two equilibria.

\section{A Distributed Algorithm}

In this section, we present an iterative and distributed algorithm for reaching the Nash equilibrium (if it exists) for the proposed power control game. The description of the algorithm is as follows.

1) Let $k=1$

2) Given the transmit powers of other users, user $k$ picks the carrier whose effective channel gain (i.e. $\hat{h}_{k \ell}$ ) is the largest and transmits only on that carrier at a power level equal to $p_{k}^{*}$

3) $k=k+1$

4) If $k \leq K$ then go back to step 2

5) Stop if the powers have converged, otherwise go to step 1

It is clear that if the above algorithm converges, it will converge to a Nash equilibrium. It is also demonstrated in
Section VII that the algorithm converges when a Nash equilibrium exists. In the case of multiple Nash equilibria, the above algorithm converges to one of the equilibria depending on the starting point.

\section{Special Case of Two Carriers}

To gain some insight into the properties of the Nash equilibria for our proposed game, let us concentrate on a system with two carriers and two users (i.e. $D=K=2$ ). We assume that the channel amplitudes are independent and identically distributed (i.i.d.) among users and carriers. If we assume Rayleigh fading (with mean 1) for the channel amplitudes then the channel gains, $h_{k \ell}$ 's, will be i.i.d. with exponential distribution of mean 1 . Let $X_{1}$ be the random variable corresponding to the number of users that transmit over the first carrier at equilibrium. If we assume $N>\gamma^{*}$, then, based on Proposition 2, the probability that both users transmit on the first carrier at equilibrium (i.e. $X_{1}=2$ ) is given by

$$
\begin{aligned}
P_{X_{1}}(2) & =\operatorname{Pr}\left\{X_{1}=2\right\} \\
& =\operatorname{Pr}\left\{\frac{h_{11}}{h_{12}}>\Theta_{2}, \frac{h_{21}}{h_{22}}>\Theta_{2}\right\}=\left(\frac{1}{1+\Theta_{2}}\right)^{2}
\end{aligned}
$$

Similarly, the probability of both users transmitting on the second carrier at equilibrium is

$$
\begin{aligned}
P_{X_{1}}(0) & =\operatorname{Pr}\left\{X_{1}=0\right\} \\
& =\operatorname{Pr}\left\{\frac{h_{12}}{h_{11}}>\Theta_{2}, \frac{h_{22}}{h_{21}}>\Theta_{2}\right\}=\left(\frac{1}{1+\Theta_{2}}\right)^{2}
\end{aligned}
$$

The probability of one user transmitting on each of the two carriers can be found to be

$$
P_{X_{1}}(1)=\operatorname{Pr}\left\{X_{1}=1\right\}=2\left(\frac{1}{1+\Theta_{0}}\right)^{2}-\left(\frac{1-\Theta_{0}}{1+\Theta_{0}}\right)^{2} .
$$

Consequently, the probability that no Nash equilibrium exists is given by

$$
\begin{aligned}
P_{o} & =\operatorname{Pr}\{\text { No Nash equilibrium }\} \\
& =2\left\{\left(\frac{\Theta_{0}}{1+\Theta_{0}}\right)^{2}-\left(\frac{1}{1+\Theta_{2}}\right)^{2}\right\} .
\end{aligned}
$$

It should be noted that as the processing gain $N$ becomes larger, $\Theta_{0}$ and $\Theta_{1}$ approach 1 from below and above, respectively. This results in a reduction in $P_{X_{1}}(1)$ but an increase in $P_{X_{1}}(0)$ and $P_{X_{1}}(2)$, i.e. the probability mass function (pmf) for $X_{1}$ becomes flatter. However, the increase outweighs the decrease and as a result $P_{o}$ decreases as $N$ increases. Going back to Fig. 1 we see that the region for which no Nash equilibrium exists shrinks as $N$ increases. In addition, the region for which more than one equilibrium exists disappears as $N$ becomes very large. Therefore, we can say that as the processing gain becomes large, the probability that the proposed power control game has a unique Nash equilibrium approaches one.

So far, the assumption has been that $N>\gamma^{*}$ so that both users can achieve $\gamma^{*}$ even when they are transmitting over the same carrier. For the case of $N \leq \gamma^{*}$, the users cannot 
achieve $\gamma^{*}$ simultaneously when they are transmitting on the same carrier and hence they would end up transmitting at the maximum power. Therefore, when $N \leq \gamma^{*}$, the probability of both users transmitting on the same carrier at equilibrium is virtually zero, i.e. $P_{X_{1}}(2)=P_{X_{1}}(0) \approx 0$. Hence, we have $P_{o}=2\left(\frac{\Theta_{0}}{1+\Theta_{0}}\right)^{2}$.

Although obtaining explicit expressions for the probabilities of the occurrence of various Nash equilibria for the case of $K>2$ is difficult, many of the statements made for the case of $K=2$ are also valid when $K>2$. Namely, as $N$ increases the pmf of $X_{1}$ becomes wider and at the same time the probability that no equilibrium exists becomes smaller. This means that in the asymptotic case of large processing gain, the proposed power control game has a unique equilibrium. Furthermore, for very large values of $N$, the pmf of $X_{1}$ can be approximated as

$P_{X_{1}}(m)=\operatorname{Pr}\left\{X_{1}=m\right\} \approx\left(\begin{array}{c}K \\ m\end{array}\right)\left(\frac{1}{2}\right)^{K}$ for $m=0, \cdots, K$.

In the next section, we show the validity of these claims using simulation.

\section{Simulation Results}

We first consider the case of two carriers with two users. We assume $L=M=100, R=100 \mathrm{Kbps}$ and $\sigma^{2}=5 \times 10^{-16}$ Watts; and use $f(\gamma)=\left(1-e^{-\gamma}\right)^{M}$ as the efficiency function. For this efficiency function, $\gamma^{*}=6.4(=8.1 \mathrm{~dB})$. We assume that the channel gains are i.i.d. with exponential distribution of mean 1 . We consider 20,000 realizations of channel gains. For each realization, we run the algorithm proposed in Section $\nabla$ for 20 iterations. If convergence is reached by the end of the $20^{t h}$ iteration, we record the number of users that transmit on each carrier; otherwise, we assume there is no equilibrium. For our simulations, $P_{\max }$ is assumed to be very large which translates to having no transmit power limit for the user terminal.

It is observed that the distributed algorithm proposed in Section $\nabla$ converges when a Nash equilibrium exists. Recall that $P_{X_{1}}(m)$ represents the probability that $m$ users transmit on the first carrier at equilibrium. Fig. 2] shows $P_{X_{1}}(2), P_{X_{1}}(1)$ and $P_{o}$ (probability of no equilibrium) as a function of the processing gain $N$. The analytical expressions obtained in Section VI] are also plotted. We see that there is a close agreement between the simulation results and the analytical values. It is also observed that as $N$ becomes large, $P_{o}$ approaches zero. For $N=16$, for example, the probability that a Nash equilibrium exists is about $93 \%$. Since $P_{X_{1}}(0)$ is identical to $P_{X_{1}}(2)$, it is not shown in the figure.

Fig. 3] shows $P_{X_{1}}(m)$ as a function of $m$ for different values of $N$. We can see from the figure that as the processing gain increases, the pmf of $X_{1}$ becomes flatter. This is because for larger values of $N$, the system becomes more tolerant towards interference. Therefore, the probability with which the two users are able to transmit on the same carrier at equilibrium increases.

We repeat the above experiment for the case of two carriers and 10 users. Fig. 4 shows $P_{X_{1}}(m)$ as a function of the

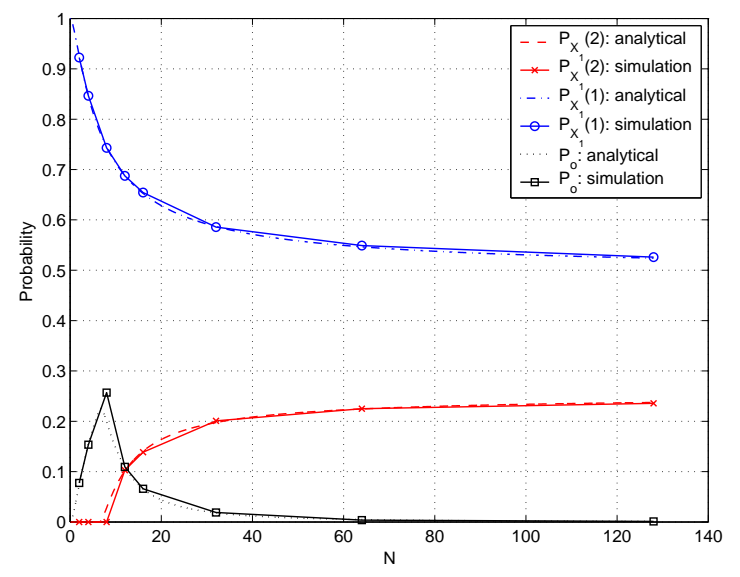

Fig. 2. Probability of having $m$ users on the first carrier at equilibrium, $P_{X_{1}}(m)$, for $m=1,2$ as well as probability of having no equilibrium are shown for $D=K=2$.

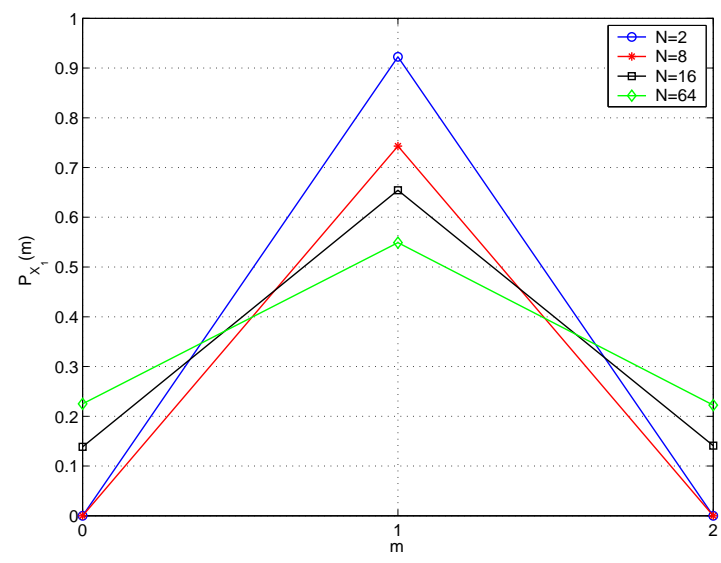

Fig. 3. Probability mass function of $X_{1}$ for different processing gains ( $D=2$ and $K=2$ )

processing gain. Due to symmetry, we have only plotted the probabilities for $m=5,6, \ldots, 10$. The probability that no equilibrium exists $\left(P_{o}\right)$ is also shown. Similar trends as those observed in the case of $K=2$ are also seen here. We observe that here again as $N$ becomes large, $P_{o}$ approaches zero. $P_{X_{1}}(m)$ as a function of $m$ for different values of $N$ is plotted in Fig. 5 The asymptotic approximation for $P_{X_{1}}(m)$ which is given by (26) is also shown. We see from the figure that as $N$ increases the pmf of $X_{1}$ becomes wider because the system becomes more interference-tolerant. Also, the equilibria for which the allocation of users to the carriers is highly asymmetric (e.g. $m=9$ and 10) are unlikely to happen. In other words, with a high probability, the users are evenly distributed between the two carriers. It should be noted that when $N$ is small, $\gamma^{*}$ cannot be achieved simultaneously by all the users at the output of the matched filters. Therefore, users keep increasing their transmit powers and hence no equilibrium is reached. This is the case until $N$ becomes large enough so that it can accommodate at least 5 users on each carrier (i.e. $N>25.6$ ). In practice, however, if $N$ is not large enough, some or all users end up transmitting at the maximum power.

We now compare the proposed approach, which jointly 


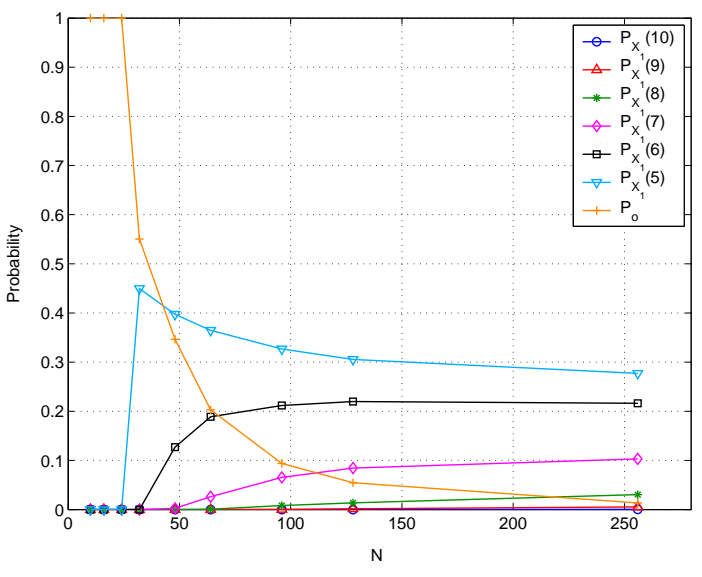

Fig. 4. Probability of having $m$ users on the first carrier at equilibrium, $P_{X_{1}}(m)$, for $m=5,6, \cdots, 10$ as well as probability of having no equilibrium are shown for $D=2$ and $K=10$.

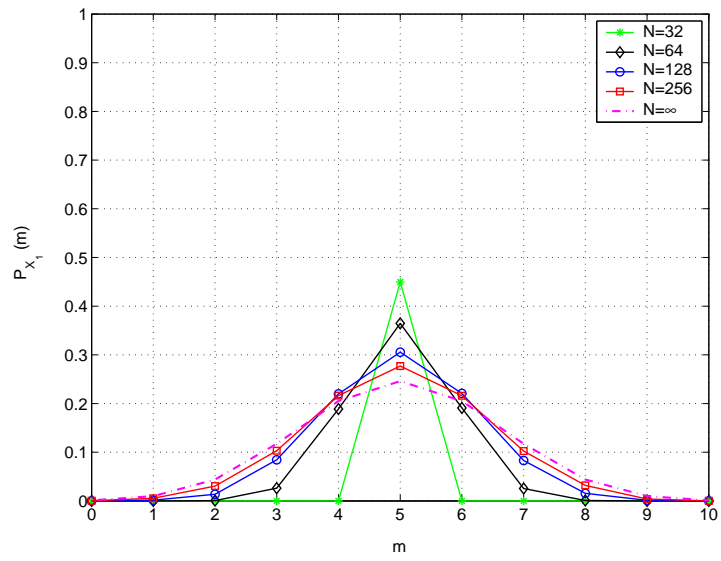

Fig. 5. Probability mass function of $X_{1}$ for different processing gains $(D=2$ and $K=10$ )

maximizes each user's utility over all carriers, with the approach that maximizes user's utility independently over each carrier. In the joint maximization approach each user transmits only on the carrier that has the best effective channel whereas in the other case, all users transmit on all the carriers such that the output SIR on each carrier is $\gamma^{*}$. We consider a system with 2 carriers and $N=128$. We fix $K$ and compute the sum of the utilities achieved by all users for 20,000 channel realizations. The utility for each case is the ratio of the total transmitted bits over the two carriers divided by the total energy consumed. Fig. 6 shows the average total utility vs. $K$ for the two approaches. We see a significant improvement in the achieved utility when joint maximization over all carriers is used. This is because when all the users transmit on every carrier, they cause unnecessary interference. To achieve $\gamma^{*}$, each user is hence forced to transmit at a higher power level which in turn results in a considerable reduction in the overall utility.

\section{CONCLUSION}

We have modelled power control for multi-carrier CDMA systems as a non-cooperative game in which each user needs to

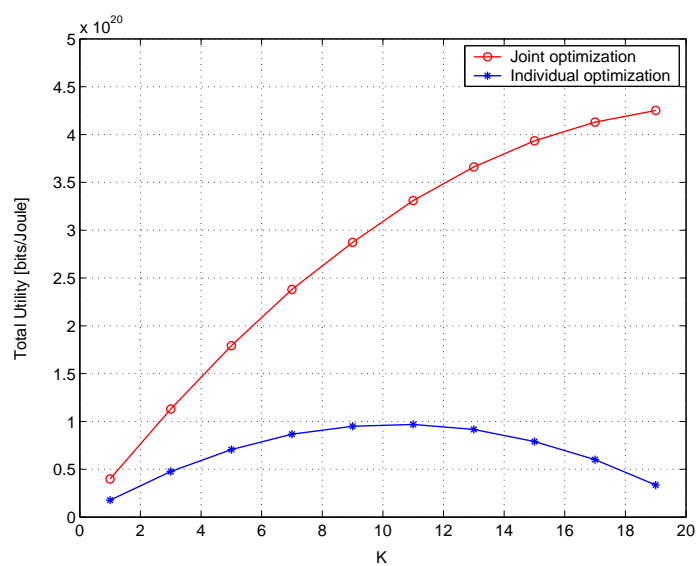

Fig. 6. Total Utility vs Number of Users ( $D=2$ and $N=128)$

decide how much power to transmit over each carrier to maximize its overall utility. The utility function has been defined as the overall throughput divided by the total transmit power over all the carriers and has units of bits per joule. For this utility function, we have shown that the utility is maximized when each user transmits only on the carrier which has the best "effective channel" for that user. In addition, we have derived the conditions for existence of a Nash equilibrium and proposed an iterative and distributed algorithm for reaching the equilibrium (if it exists). The properties of the Nash equilibria for the proposed game have been studied. We have also demonstrated the performance improvement resulting from the proposed approach compared to the approach of independent optimization over each carrier.

\section{REFERENCES}

[1] D. J. Goodman and N. B. Mandayam, "Power control for wireless data," IEEE Pers. Commun., vol. 7, pp. 48-54, April 2000.

[2] M. Xiao, N. B. Shroff, and E. K. P. Chong, "Utility-based power control in cellular wireless systems," Proc. of the Annual Joint Conf. of the IEEE Computer and Communications Societies (INFOCOM), pp. 412421, 2001. Alaska.

[3] C. U. Saraydar, N. B. Mandayam, and D. J. Goodman, "Efficient power control via pricing in wireless data networks," IEEE Trans. Commun., vol. 50, pp. 291-303, February 2002.

[4] F. Meshkati, H. V. Poor, S. C. Schwartz, and N. B. Mandayam, "Linear multiuser receivers and power control in wireless data networks: A gametheoretic approach," Proc. of the $37^{\text {th }}$ Annual Conf. on Information Sciences and Systems (CISS), March 2003. Baltimore, MD.

[5] F. Meshkati, H. V. Poor, S. C. Schwartz, and N. B. Mandayam, "A gametheoretic appraoch to power control and receiver design in wireless data networks with multiple antennas," Proc. of the $41^{\text {st }}$ Annual Allerton Conf. on Communication, Control, and Computing, October 2003. Monticello, IL.

[6] F. Meshkati, H. V. Poor, S. C. Schwartz, and N. B. Mandayam, "A utilitybased appraoch to power control and receiver design in wireless data networks." Submitted to the IEEE Trans. Commun.

[7] S. Hara and R. Prasad, "Overview of multicarrier CDMA," IEEE Commun. Mag., pp. 126-133, December 1997.

[8] D. J. Goodman and N. B. Mandayam, "Network assisted power control for wireless data," Proc. of the IEEE 53 ${ }^{\text {rd }}$ Vehicular Technology Conference, pp. 1022-1026, Spring 2001. Rhodes, Greece.

[9] W. Yu, G. Ginis, and J. M. Cioffi, "Distributed multiuser power control for digital subscriber lines," IEEE J. Select. Areas Commun., vol. 20, pp. 1105-1115, June 2002. 